EPJ manuscript No.

(will be inserted by the editor)

\title{
Multiplicity distributions inside parton cascades developing in a medium
}

\author{
Nicolas Borghini ${ }^{1}$ \\ CERN, Department of Physics, Theory Division, CH-1211 Genève 23, Switzerland \\ Received: date / Revised version: date
}

\begin{abstract}
The explanation of the suppression of high- $p_{T}$ hadron yields at RHIC in terms of jet-quenching implies that the multiplicity distributions of particles inside a jet and jet-like particle correlations differ strongly in nucleus-nucleus collisions at RHIC or at the LHC from those observed at $e^{+} e^{-}$or hadron colliders. We present a framework for describing the medium-induced modification, which has a direct interpretation in terms of a probabilistic medium-modified parton cascade, and which treats leading and subleading partons on an equal footing. We show that our approach can account for the strong suppression of single inclusive hadron spectra measured in $\mathrm{Au}-\mathrm{Au}$ collisions at $\mathrm{RHIC}$, and that this implies a characteristic distortion of the single inclusive distribution of soft partons inside the jet. We determine, as a function of the jet energy, to what extent the soft fragments within a jet can be measured above some momentum cut.
\end{abstract}

PACS. 12.38.Mh Quark-gluon plasma - 25.75.-q Relativistic heavy-ion collisions

\section{Introduction}

Among the most notable results from the first years of nucleus-nucleus collisions at RHIC stand the deficit in high transverse-momentum hadrons and the suppression ' of leading back-to-back hadron correlations observed in central $\mathrm{Au}-\mathrm{Au}$ collisions with respect to expectations from scaling the yields measured in $p p$ or $\mathrm{d}-\mathrm{Au}$ collisions [1]. These observations are consistent with the "jet-quenching" scenario: before they hadronize in the vacuum, partons produced in the dense matter created in head-on $\mathrm{Au}-\mathrm{Au}$ collisions dissipate a significant fraction of their energy through an enhanced radiation of soft gluons 2, 3,4].

Irrespective of the details of the implementation of the medium-enhanced radiation of gluons - either through coherent multiple soft-momentum transfers à la LandauPomeranchuk-Migdal [5],6], or through single hard scattering [3 — jet-quenching models of inelastic (radiative) energy loss are quite successful in explaining present lighthadron data from RHIC [7,8,9]. Nevertheless, there remains much room for technical improvement over the existing formulations of inelastic energy loss. Thus, a generic feature of these approaches is that they only consider the medium-induced enhancement in gluon radiation for the leading parton, discarding the medium influence on subleading partons. Such an approximation may remain under control when dealing with leading-hadron production; however, predictions involving subleading particles become questionable, be it for jet shapes, which may become experimentally accessible at the LHC [10], or simply for intrajet two-particle correlations. Similarly, in exist- ing models energy-momentum conservation is not explicitly conserved at each parton splitting, but only globally, through various ad hoc corrections.

A novel formulation of medium-induced parton energy loss was recently introduced in Ref. [1], which aims at correcting some of the shortcomings of standard approaches mentioned above. Thus, it is the first one that deals equally with the various splittings of both leading and subleading partons inside a shower. Furthermore, it automatically conserves energy-momentum at each parton splitting. These improvements are admittedly obtained, at the moment, at the cost of a less accurate treatment of other aspects of radiative energy loss, which should be restored in a future Monte-Carlo implementation. Nevertheless, the physical constraints we choose to emphasize in our approach may prove to be of greater importance than those retained in other existing models, in particular for the discussion of high- $p_{T}$ particle correlations (see, however, Refs. [12,13] for an alternative extension of parton energy loss which allows one to tackle that issue) or multiplicity distributions inside jets.

\section{Formalism}

One of the most testing ground of the color structure of quantum chromodynamics (QCD) is provided by the jets that are created in elementary-particle collisions, be it in $e^{+} e^{-}$or in $p p / p \bar{p}$ collisions. Thus, the asymptotic shape of the distribution of hadron momenta inside a jet can be computed exactly, especially at small momentum frac- 
tions $x=p / E_{\text {jet}}$, by a resummation of infrared-singular terms to all orders, within the so-called Modified Leading Logarithmic Approximation (MLLA) of QCD [14,15. 16. Color coherence thus results in destructive interference between partons, which leads to a suppression of hadrons with small $x$. The interference is actually equivalent, to double and single logarithmic accuracy in $\ln (1 / x)$ and $\ln \left(Q / \Lambda_{\text {eff }}\right)$ - where $Q \sim E_{\text {jet }}$ is the jet virtuality and $\Lambda_{\text {eff }}$ an infrared cutoff which is eventually fitted to experimental data - to an angular ordering of the sequential parton decays within the shower, with leadingorder splitting functions. An important prediction of this angular-ordered probabilistic parton cascade is, to nextto-leading order $\sqrt{\alpha_{S}}$, the characteristic "hump-backed plateau" shape of the distribution of parton momenta inside a jet, represented as a function of $\ln (1 / x)$. The parton shower, evolved down to an infrared cutoff $Q_{0} \sim \Lambda_{\text {eff }}$, is eventually identified to a hadron jet, by mapping locally each parton onto a hadron ("Local Parton-Hadron Duality", LPHD): for each hadron type, the hadron distribution equals $K^{h}$ times the parton distribution, where $K^{h}$ is a proportionality factor of order unity. ${ }^{1}$ This resummation and the LPHD prescription give a good description of the measured longitudinal distributions of hadrons $D^{h}\left(x, Q^{2}\right)$ over a wide energy range, both in $e^{+} e^{-}$[17.18 and in $p \bar{p}$ [19] collisions. For instance, Fig. 1] shows the distribution of inclusive hadrons $D^{h}\left(x, Q^{2}\right)$ inside $17.5 \mathrm{GeV}$ jets, a typical jet energy at RHIC, as measured in $e^{+} e^{-}$annihilation by the TASSO Collaboration [17, together with the MLLA prediction with $K^{h}=1.35$.

The formalism developed in Ref. 11] to describe the medium-induced distortion of jets reduces to the MLLA baseline in the absence of a medium. This new approach involves different approximations, emphasizing other physical aspects, from the standard models of parton energy loss that are currently used in the phenomenology of RHIC data. Thus, present model comparisons to RHIC data start with a medium-modified energy spectrum of radiated gluons, $\mathrm{d} I^{\text {tot }}=\mathrm{d} I^{\mathrm{vac}}+\mathrm{d} I^{\text {med }}$ 2,3 4. The part corresponding to the "normal" vacuum radiation shows a double logarithmic dependence $\mathrm{d} I^{\mathrm{vac}}=\frac{\alpha_{s}}{\pi^{2}} \frac{\mathrm{d} \omega}{\omega} \frac{\mathrm{dk}}{\mathbf{k}^{2}}$; its integral over $\mathbf{k}$ gives rise to the leading $\ln Q^{2}$ term in the DGLAP evolution equation. This contrasts to the $\mathbf{k}$-integration of $\mathrm{d} I^{\mathrm{med}}$, which is infrared- and ultraviolet-safe 6 and leads to a nuclear-enhanced "higher-twist" contribution, $\propto \hat{q} L / Q^{2}$, where $\hat{q}$ is the transport coefficient that characterizes the medium, subleading in an expansion in $1 / Q^{2}$, but enhanced with respect to other such terms by a factor proportional to the geometrical extension $\sim L$ of the

1 In addition to using different phenomenological proportionality constants $K^{h}$, the hadron-type dependence in MLLA is also implemented by cutting the development of the parton cascade at different scales $Q_{0}$, with $Q_{0}$ chosen equal to the hadron mass. This requires using a slightly more elaborate form of the spectrum than Eq. (1), see Ref. [16], and leads to an energy-independent stiffening of spectra for heavier hadrons. The comparison to inclusive yields in elementary-particle collisions were done with a unique cutoff parameter for all particle types, $Q_{0}=\Lambda_{\text {eff }}=253 \mathrm{MeV}$.

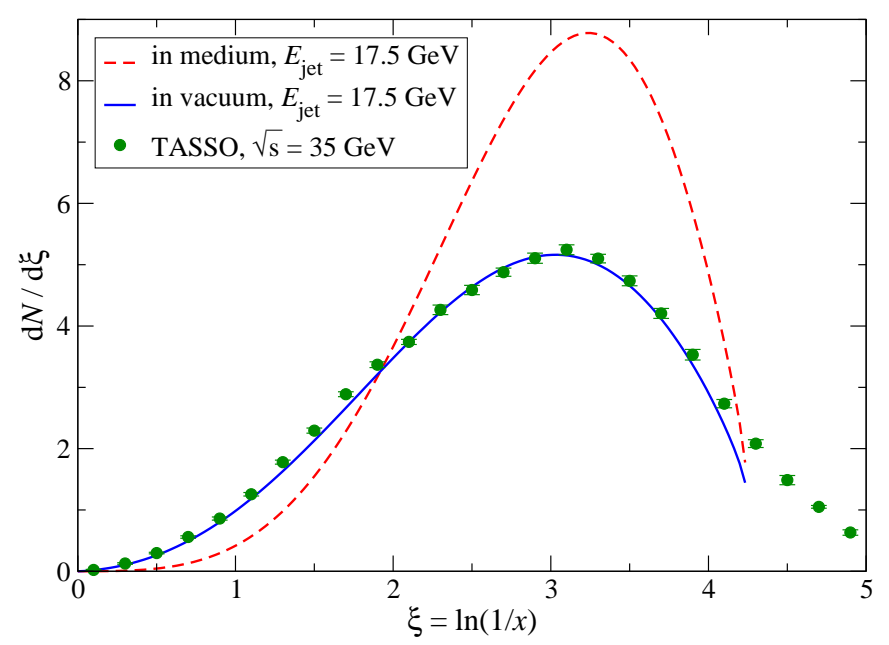

Fig. 1. Longitudinal distribution $\mathrm{d} N / \mathrm{d} \ln (1 / x)$ of inclusive hadrons inside a jet of energy $E_{\text {jet }}=17.5 \mathrm{GeV}$, as a function of $\ln (1 / x)=\ln \left(E_{\text {jet }} / p\right)$, as measured by TASSO [17] and within MLLA (solid curve: $f_{\text {med }}=0$; dashed curve: $f_{\text {med }}=0.8$ ).

target. In practice, however, the parton virtuality does not enter the existing comparisons to experimental data, where one rather considers the $\mathbf{k}$-integrated gluon distribution $\omega \frac{\mathrm{d} I^{\text {med }}}{\mathrm{d} \omega}$, neglecting the $Q^{2}$-dependence. In addition, existing approximations only include the extra source of gluon radiation $\mathrm{d} I^{\text {med }}$ for the leading parton, dropping it for the further medium-induced splittings of subleading partons in the shower.

The obvious way to improve over this state of the art is to replace the double differential gluon spectrum $\mathrm{d} I^{\mathrm{vac}}$ by $\mathrm{d} I^{\text {tot }}$ in all leading and subleading splitting processes of a medium-modified parton cascade. This can only be done within a Monte-Carlo approach, which we intend to develop in future studies. The first step in that direction, which is still fully analytical, consists in using an additional approximation: instead of using the computed k-integrated medium-induced distribution $\omega \frac{\mathrm{d} I^{\text {med }}}{\mathrm{d} \omega}$, we replaced it by a constant $f_{\text {med }}$. One can check that this is not too crude an assumption in the kinematic regime tested at RHIC, where it amounts to a similar uncertainty as that arising from whether one should use the multiplesoft scattering approach or the single hard scattering picture. Under this assumption, we have then used the extra medium-induced spectrum $\omega \frac{\mathrm{d} I^{\text {med }}}{\mathrm{d} \omega}$ on the same level as $\omega \frac{\mathrm{d} I^{\mathrm{vac}}}{\mathrm{d} \omega}$, i.e., as a leading logarithmic correction [11. With this ansatz, our formalism ensures energy-momentum conservation at each parton splitting, and treats all leading and subleading parton splittings on the same footing.

\section{Phenomenological predictions}

As stated above, in our analytical approach we approximate the medium-induced contribution to the gluon spectrum $\omega \frac{\mathrm{d} I^{\mathrm{med}}}{\mathrm{d} \omega}$ by a constant $f_{\text {med }}$ in the kinematically relevant range of $\omega$. This actually amounts to considering 
"medium-modified parton splitting functions", which differ from the standard ones by enhancing their singular parts by a factor $\left(1+f_{\text {med }}\right) .^{2}$ This formulation allows us to follow the same line of technical arguments as that used for the calculation of jet multiplicity distributions in the absence of a medium [16].

\subsection{Distortion of the hump-backed plateau}

We can thus compute the momentum distribution of partons within a (gluon-initiated) parton cascade:

$$
\begin{aligned}
x \bar{D}_{g}(x, \tau)= & \frac{4 N_{c}\left(1+f_{\mathrm{med}}\right) \tau}{b \hat{B}(\hat{B}+1)} \\
& \times \int_{-\mathrm{i} \infty}^{+\mathrm{i} \infty} \frac{\mathrm{d} \nu}{2 \pi \mathrm{i}} x^{-\nu} \Phi(-\hat{A}+\hat{B}+1, \hat{B}+2 ;-\nu \tau),
\end{aligned}
$$

where $\tau \equiv \ln \left(Q / \Lambda_{\text {eff }}\right), \Phi$ is the confluent hypergeometric function,

$$
\begin{aligned}
& \hat{A} \equiv \frac{4 N_{c}\left(1+f_{\text {med }}\right)}{b \nu}, \quad \hat{B}=\frac{\hat{a}}{b}, \\
& \hat{a}=\frac{11+12 f_{\mathrm{med}}}{3} N_{c}+\frac{2}{3} \frac{N_{f}}{N_{c}^{2}}, \quad b=\frac{11}{3} N_{c}-\frac{2}{3} N_{f},
\end{aligned}
$$

and $N_{f}$ is the number of active flavors. Quite naturally, by setting $f_{\text {med }}=0$ in Eq. (1) one recovers the usual "vacuum" expression of $x \bar{D}_{g}(x, \tau)$.

To exemplify the effect of the medium-enhanced gluon radiation on the hump-backed plateau of particle production, we compare in Fig. 10 the spectra Eq. (11) for a jet with energy $E_{\text {jet }}=17.5 \mathrm{GeV}$, shown as a function of $\ln (1 / x)$, in the cases $f_{\text {med }}=0$ (no medium) and $f_{\text {med }}=0.8$, where the choice of the latter value will be justified in Sec. $3.3{ }^{3}$ One clearly sees that the effect of the medium is a strong distortion of the distribution, with a depletion of the number of particles at large $x$, and correspondingly a largely enhanced emission of particles at small $x$ : due to energymomentum conservation in the parton cascade, the energy which in the vacuum is taken by a single large- $x$ parton is redistributed over many small- $x$ partons in the presence of a medium.

An alternative way to illustrate the influence of the medium on the spectra is to display the distributions as a function of the momentum fraction $x$, see Fig. 2. The plot emphasizes the suppression in high- $x$ hadron production, which is the aspect tested by experimental studies of the high- $p_{T}$ spectra of hadrons, rather than the enhancement in small- $x$ particles.

\footnotetext{
${ }^{2}$ Such a modification of parton splitting functions was discussed in Ref. [20, where it results from considering nuclearenhanced twist-four parton matrix elements in studies of deeply inelastic $e A$ scattering.

${ }^{3}$ Both spectra were actually scaled by a factor $K^{h}=1.35$, so that the vacuum one match the corresponding TASSO data. This goes along the idea that the medium affects the development of the parton shower, while hadronization takes place "in the vacuum" and thus the LPHD coefficient should be the same in both cases.
}

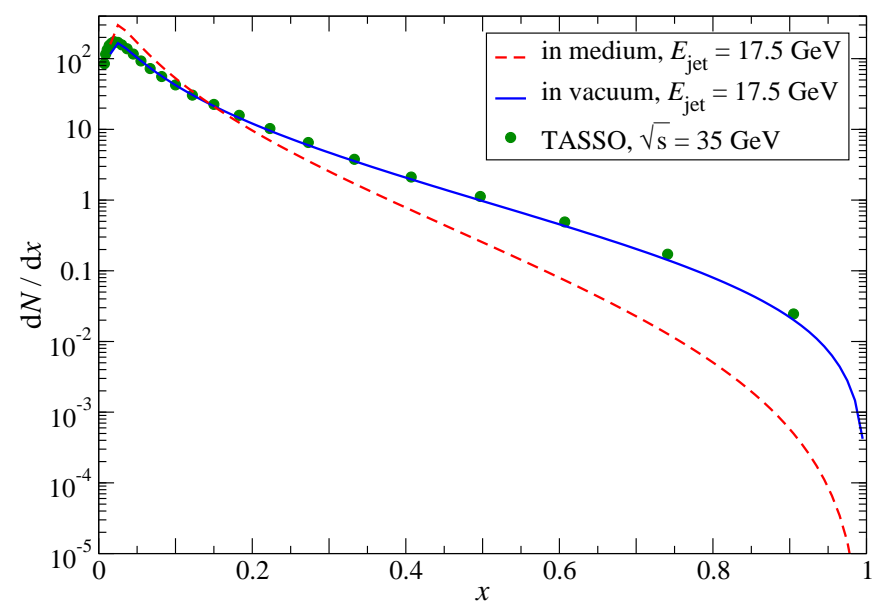

Fig. 2. Single inclusive distribution of hadrons, $\mathrm{d} N / \mathrm{d} x$, inside a jet of energy $E_{\text {jet }}=17.5 \mathrm{GeV}$, as a function of $x=E_{\text {jet }}$, as measured by TASSO [17 and within MLLA (solid curve: $f_{\text {med }}=0$; dashed curve: $f_{\text {med }}=0.8$ ).

\subsection{Associated multiplicity}

Once the longitudinal multiplicity distribution inside a jet is known, a straightforward integration yields the number of hadrons inside the jet with transverse momenta larger than a given cut, $\mathcal{N}\left(p_{T} \geq p_{T}^{\text {cut }}\right)$. We can then calculate this multiplicity for jets with the same energy both in the presence of medium effects (in which case, the lower cut gives some control on the high-multiplicity soft background of nucleus-nucleus collisions at RHIC or LHC, over which the jet develops) and in vacuum, and compute their ratio. We display the latter in Fig. 3 for jets with $E_{\text {jet }}=17.5 \mathrm{GeV}$ and medium effects modeled by a constant coefficient $f_{\text {med }}=0.8$. The ratio is seen to be smaller than 1 for $p_{T}^{\text {cut }} \gtrsim 1.5 \mathrm{GeV} / c$, while the medium-induced enhancement in soft-particle production becomes dominant for smaller values of the transverse-momentum cut. The crossover value is close to that reported by the STAR Collaboration in attempts at measuring the excess of particles inside the back jet over the soft background [21].

A more thorough comparison between our calculation and the STAR data would have to take into account several effects which we did not consider in our simple approach. First, in our calculation the energy of the jet is known and fixed, while experimentally it spans some range in energy. In the same line, we did not try to reproduce the experimental fact that the high- $p_{T}$ particles measured at RHIC come from jets that have traversed different in-medium path lengths, and thus radiated different energy fractions. Eventually, we did not model the soft background of $\mathrm{Au}-\mathrm{Au}$ collisions at RHIC, which extends well over the region where the medium-enhanced part of the multiplicity lies. Nevertheless, the reasonable agreement between our calculation and the experimental measurement is a further hint that energy is indeed redistributed from high- to low- $x$ partons through the influence of the medium, although nothing definitive on the actual shape of the distorted spectrum of radiated partons can 
Nicolas Borghini: Multiplicity distributions inside parton cascades developing in a medium

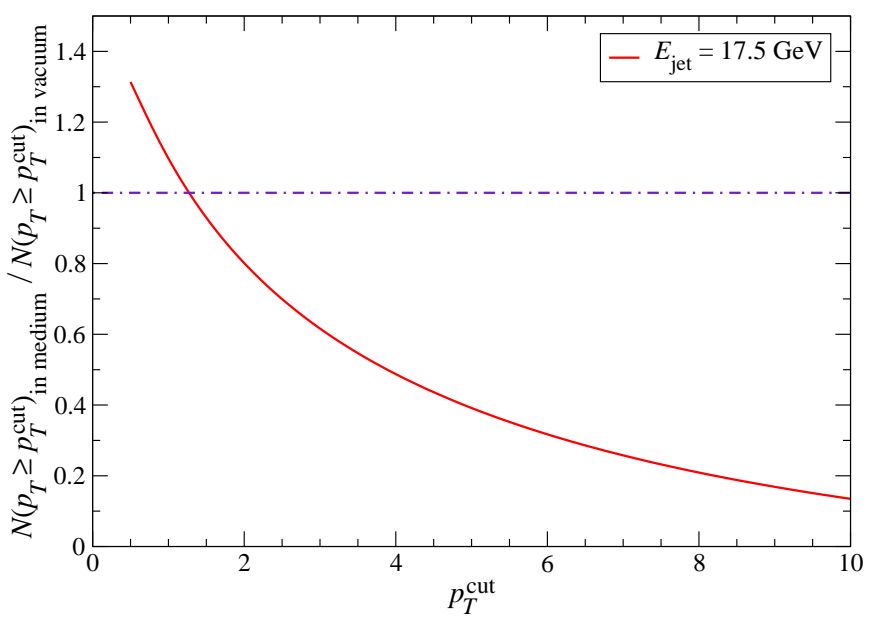

Fig. 3. Ratio of the multiplicities of particles above a lower momentum cut $p_{T}^{\text {cut }}$ inside a jets of energy $\mathrm{s} E_{\text {jet }}=17.5 \mathrm{GeV}$ developing in a medium $\left(f_{\text {med }}=0.8\right)$ and in the vacuum.

be claimed at the moment. Note, however, that the calculations reported in Ref. 11 indicate that the crossover between enhancement and depletion should take place at transverse momenta $p_{T}^{\text {cut }} \sim 4-7 \mathrm{GeV} / c$ for jets of energy $E_{\text {jet }}=100-200 \mathrm{GeV}$, which should be accessible at the LHC. This should leave a window above the upper kinematic boundary of the soft background, in which there is an enhancement of the jet multiplicity, thereby allowing a more detailed characterization of the medium-enhanced radiation.

\subsection{Nuclear modification factor}

As a last phenomenological prediction of our model, let us compute the medium-modification factor $R_{A A}$, defined as the ratio of the hadron yield in nucleus-nucleus over that in $p p$ collisions, scaled by the number of binary collisions. For that purpose, we need to supplement a further ingredient to our approach, namely the underlying partonic momentum distribution at RHIC energies. Since we only want to show that our implementation of radiative parton energy loss is able to result in a factor of 5 suppression of the hadron yields, as measured experimentally, we shall restrict ourselves to using a power-law parton spectrum $1 / p_{T}^{7}$. We do not take into account the kinematicboundary influence on the shape of the $p_{T}$-spectrum, nor "initial-state" effects like shadowing or Cronin effect. Eventually, as in Sec. 3.2 we do not attempt to model the geometry of the medium, nor the fluctuations in the energy radiated by partons with a given energy.

The result of our calculation is shown in Fig. [t together with the PHENIX $\pi^{0}$ data at $\sqrt{s_{N N}}=200 \mathrm{GeV}$ [22]. We see that our choice of $f_{\text {med }}=0.8$ for the coefficient that models medium effects allows us to reproduce a modification factor of $\sim 0.2$, which justifies our using it in all applications reported here. This suppression by a factor 5 actually reflects the observation one can make on Fig. 2 that the spectrum of radiated partons in a medium is a

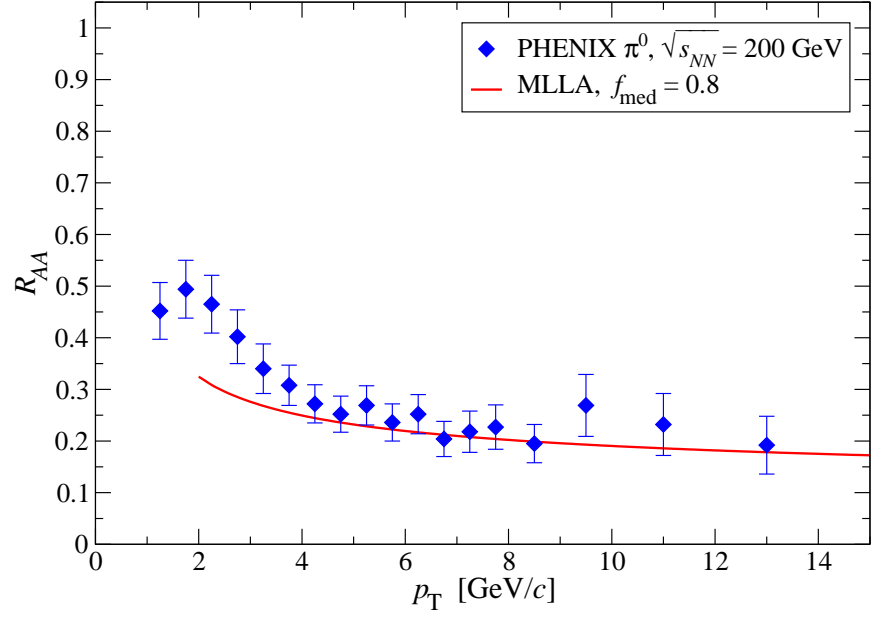

Fig. 4. Transverse-momentum dependence of the nuclear modification factor $R_{A A}$, computed for a medium-enhanced radiation of gluons modeled by $f_{\text {med }}=0.8$, compared to PHENIX data on $\pi^{0}$ production 22

factor $\sim 3-6$ times smaller than that of partons within a jet in vacuum for momentum fractions $x \sim 0.4-0.6$, where $R_{A A}$ mostly tests the spectra. Such values of $x$ are often considered to lie outside the small- $x$ region where MLLA is a proper approach to hadron production in elementaryparticle collisions. Nevertheless, the possible uncertainty of using such a resummation of large $\ln (1 / x)$ terms (instead of, for instance, fitted fragmentation functions) in computing the nuclear modification factor is relatively small, inasmuch as $R_{A A}$ is a ratio, which does not depend on the overall normalization of the vacuum spectrum, and that we are discussing a suppression by a factor of 5 , not an effect of a few percent.

\section{Conclusion}

We have reported a first step towards a description of parton cascades developing in a medium, which conserves energy-momentum at each successive parton splitting, and treats all partons in the shower on the same footing 11 . The simplified analytical formalism we have presented is able to reproduce at least semi-quantitatively several characteristic features of RHIC data, such as the suppression of high-momentum particle yields and the enhanced softparticle distribution associated to high- $p_{T}$ trigger particles. The price we pay for an analytical treatment is the inaccurate handling of the $Q^{2}$-dependence of parton radiation, which is not dealt with more properly in other existing formalisms of radiative parton energy loss, and the approximate treatment of the $\omega$-dependence of the spectrum, which might not be of prime importance in the RHIC regime.

In the future, we expect to remove the latter deficiencies by implementing our approach within a Monte-Carlo formulation. In that respect, the analytical results presented here will serve as a powerful consistency check for the correct implementation of splitting processes in the 
Monte-Carlo simulation. Such simulations will allow us to address the issue whether and how medium-induced parton energy loss depends on virtuality, as heuristic arguments suggest it should: since a hard parton of virtuality $Q$ and energy $E$ has a lifetime $\sim E / Q^{2}$ in the rest frame of the dense matter through which it propagates, before it branches, high-virtuality partons will branch so quickly that their splitting is not influenced by the presence of the medium (so that $f_{\text {med }}$ should decrease with increasing $\left.Q^{2}\right)$. This question will be particularly important for addressing the logarithmically wide kinematic range accessible in $\mathrm{Pb}-\mathrm{Pb}$ collisions at the LHC. An angular-ordered Monte-Carlo parton cascade will also shed light on other issues that have been studied in the absence of medium effects, as two-particle correlations within jets [15.23 24, transverse jet broadening, or the differences between lightand heavy-quark jets 25]. Finally, it will also allow one to implement realistic features of the collision, as e.g. fluctuations or the geometry of the dense created medium traversed by the fast parton.

Acknowlegements. Fruitful discussions and collaboration with Urs Wiedemann are warmly acknowledged.

\section{References}

1. See P. Jacobs and X.-N. Wang, Prog. Part. Nucl. Phys. 54 (2005) 443, and references therein.

2. R. Baier, D. Schiff and B. G. Zakharov, Ann. Rev. Nucl. Part. Sci. 50 (2000) 37.

3. M. Gyulassy, I. Vitev, X.-N. Wang and B. W. Zhang, in Quark Gluon Plasma III (World Scientific, Singapore, 2004), p. 123.

4. A. Kovner and U. A. Wiedemann, in Quark Gluon Plasma III (World Scientific, Singapore, 2004), p. 192.

5. R. Baier, Yu. L. Dokshitzer, A. H. Mueller, S. Peigné and D. Schiff, Nucl. Phys. B 484 (1997) 265.

6. C. A. Salgado and U. A. Wiedemann, Phys. Rev. D 68 (2003) 014008.

7. S. Turbide, C. Gale, S. Jeon and G. D. Moore, Phys. Rev. C 72 (2005) 014906.

8. A. Dainese, C. Loizides and G. Paic, Eur. Phys. J. C 38 (2005) 461.

9. K. J. Eskola, H. Honkanen, C. A. Salgado and U. A. Wiedemann, Nucl. Phys. A 747 (2005) 511.

10. M. López-Noriega, these proceedings.

11. N. Borghini and U. A. Wiedemann, hep-ph/0506218 hep-ph/0509364

12. A. Majumder and X.-N. Wang, Phys. Rev. D 70 (2004) 014007.

13. A. Majumder, E. Wang and X.-N. Wang, nucl-th/0412061

14. A. H. Mueller, Nucl. Phys. B 213 (1983) 85.

15. A. Bassetto, M. Ciafaloni and G. Marchesini, Phys. Rep. 100 (1983) 201.

16. Yu. L. Dokshitzer, V. A. Khoze and S. I. Troian, Adv. Ser. Direct. High Energy Phys. 5 (1988) 241.

17. W. Braunschweig et al. [TASSO Collaboration], Z. Phys. C 47 (1990) 187.

18. G. Abbiendi et al. [OPAL Collaboration], Eur. Phys. J. C 27 (2003) 467.
19. D. Acosta et al. [CDF Collaboration], Phys. Rev. D 68, 012003 (2003).

20. X. F. Guo and X. N. Wang, Phys. Rev. Lett. 85 (2000) 3591.

21. J. Adams et al. [STAR Collaboration], Phys. Rev. Lett. 95 (2005) 152301.

22. S. S. Adler et al. [PHENIX Collaboration], Phys. Rev. Lett. 96 (2006) 202301.

23. C. P. Fong and B. R. Webber, Phys. Lett. B 241 (1990) 255.

24. R. Perez-Ramos, JHEP 0606 (2006) 019; hep-ph/0607223

25. Yu. L. Dokshitzer, F. Fabbri, V. A. Khoze and W. Ochs, Eur. Phys. J. C 45 (2006) 387. 